\title{
DESIGN OF S-BAND CAVITY BPM FOR HLS *
}

\author{
Q. Luo", B. G. Sun, D. H. He, P. Li, J. H. Wang, P. Lu \\ NSRL, University of Science and Technology of China, Hefei 230029, P. R. China
}

\begin{abstract}
For the development of accelerators we require increasingly precise control of beam position. Cavity BPMs promise a much higher position resolution compared to other BPM types and manufacture of cavity BPMs is in general less complicated. The cavity BPM operating at S-band for HLS (Hefei Light Source) was designed. It consists of two cavities: a position cavity tuned to TM110 mode and a reference cavity tuned to TM010 mode. To suppress the monopole modes we use waveguides as pickups. Superheterodyne receivers are used in electronics for many cavity BPMs while we decide to use chip AD8302 produced by Analog Devices to process the signals. To simulate and calculate the electromagnetic field we use MAFIA.
\end{abstract}

\section{INTRODUCTION}

We have a plan to develop a new injector for HLS, thus an improvement of the beam position monitor system is required. To meet the demand in resolution of the Photocathode RF Gun for new injector design we decided to use cavity BPM instead of the stripline and button BPMs we used in HLS ${ }^{[1]}$ because cavity beam position monitors have relatively higher resolution compared to those old designs ${ }^{[2-4]}$. Cavity BPM uses a cylindrical cavity mounted into the beam-pipe as pick-up station. When an off-centered beam passes through the cavity it excites electromagnetic field, including TM110 mode. Signal of TM110 mode in the beam-pipe region has a linear dependence on the bunch displacement, so we can use it for beam position monitoring ${ }^{[5]}$. The TM010 signal is many orders of magnitude larger than the TM110 signal as the beam offset is very small, so we use waveguides as pickups to suppress the TM010 signal and increase the precision ${ }^{[6]}$. We use electronics for signal detection and computation, then analyze and display the results on computer ${ }^{[7]}$. As it is mentioned before, the signal of TM110 mode in the beam-pipe region has a linear dependence of the value of the bunch displacement, to get the position of the bunch we still have to get a signal which gives the phase of the displacement, a reference cavity is then developed to give this signal. Though superheterodyne circuits are generally used in signal processing for cavity BPMs, we use chip AD8302 produced by Analog Devices to process the signals. AD8302 can work out the amplitude ratio and phase separation of two signals, so we can use it to process the signals from position cavity and reference cavity and then give the exact position of the beam.

\footnotetext{
* Supported by the National "985 Project" (173123200402002)

\# luoqing@mail.ustc.edu.cn
}

06 Instrumentation, Controls, Feedback \& Operational Aspects

\section{S-BAND CAVITY AND WAVEGUIDE COUPLER}

The coupling device could be antennae or waveguides. For higher frequency the antennae may be too tiny, and for lower frequency the size of waveguides may be too large. As the beam offset is very small, the TM010 signal is many orders of magnitude larger than the TM110 signal. We can not use the signal from one pickup to determine the beam position directly. Usual practice is to use a magic-T to subtract the signal of TM010 mode. Another way is to use H-plane of waveguides to couple magnetically to the side of the cavity and then suppress the TM010 mode, and the signal can then be directly used to obtain beam position. This way provides looser tolerances and simplifies the manufacture of the BPM system. The new BPM system will be put into service with Photocathode RF Gun for new injector, so we should select a working frequency of the position cavity around our injector RF frequency, $2856 \mathrm{MHz}$. Since there's heavy interference at $2856 \mathrm{MHz}$ and the circuit chip we used has a upper measuring limit of $2.7 \mathrm{GHz}$, we finally decided to use a working frequency around $2652 \mathrm{MHz}$. We do not have to use a frequency exactly at $2856 \mathrm{MHz}$ because the BPM system will be used in single bunch case and the frequency spectrum is continuous. Given these conditions we chose waveguides as pickups.

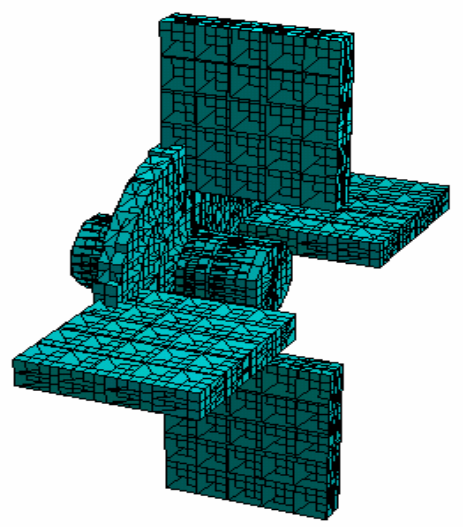

Figure 1: Pattern of the pick-up station

Fig.1 shows the pattern of the pick-up station of the cavity BPM system using MAFIA, the position cavity. A beam pipe passes through the centre of the cavity which four waveguide couplers are installed into it. The parameters of the pick-up station are in Table 1('Distance' is the distance between the bottom of the waveguide and the axis of the cavity, and we set the length of the beam pipe to $80 \mathrm{~mm}$ ): 
Table 1: Parameters of the pick-up station

\begin{tabular}{|l|l|}
\hline Cavity radius & $65 \mathrm{~mm}$ \\
\hline Beam pipe Radius & $35 \mathrm{~mm}$ \\
\hline Cavity length & $10 \mathrm{~mm}$ \\
\hline Distance & $43 \mathrm{~mm}$ \\
\hline Frequency of TM010 & $1847.2 \mathrm{MHz}$ \\
\hline Frequency of TM110 & $2651.3 \mathrm{MHz}$ \\
\hline Q0 of TM110 & 7948.5 \\
\hline
\end{tabular}

Waveguides we used have parameters showed in table 2 as pickups. The work frequency of the waveguides can be from $2.50 \mathrm{GHz}$ to $3.80 \mathrm{GHz}$.

Table 2: Parameters of the waveguides

\begin{tabular}{|l|l|}
\hline Waveguide a & $75.0 \mathrm{~mm}$ \\
\hline Waveguide b & $12.9 \mathrm{~mm}$ \\
\hline Waveguide length & $82.0 \mathrm{~mm}$ \\
\hline
\end{tabular}

\section{Cavity Radius and Waveguides Adjustments}

In actual computation we found that the variation of the TM110 frequency caused by the change of the cavity radius is very small, much less than variation caused by the change of waveguide length and the distance between the waveguide and the axis. The variation of the TM010 frequency caused by the change of the radius is obvious and much larger than that caused by the waveguide adjustment. Fig.2-4 below shows the detail result when the cavity length is set to $40 \mathrm{~mm}$.

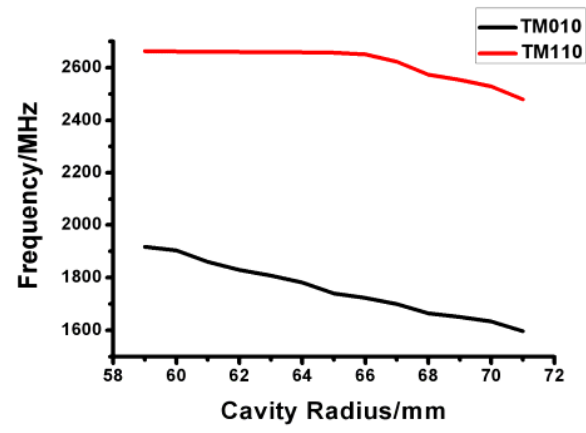

Figure 2: Effect of cavity radius

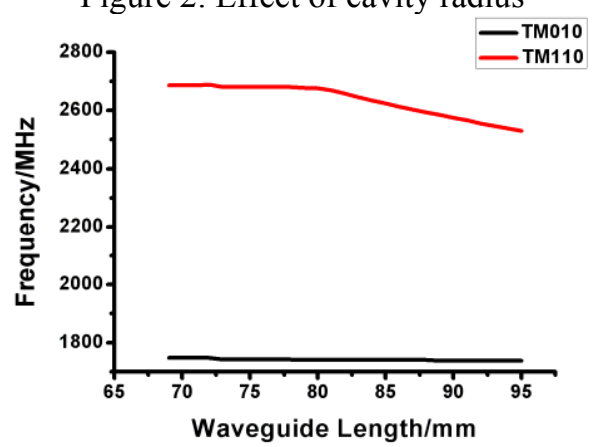

Figure 3: Effect of waveguide length

06 Instrumentation, Controls, Feedback \& Operational Aspects

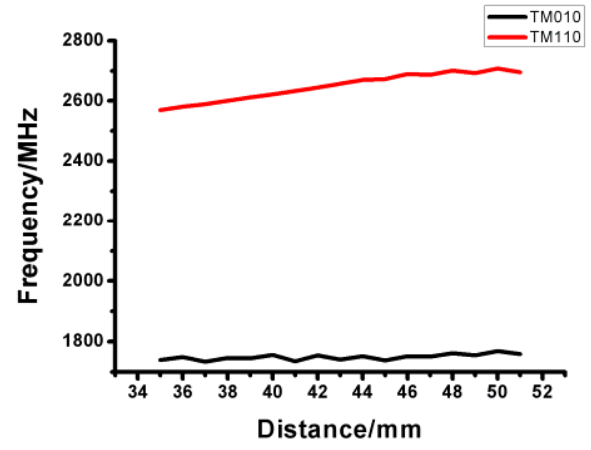

Figure 4: Effect of distance

\section{Effect of Cavity Length}

In Fig.2-4 the cavity length is set to $40 \mathrm{~mm}$, in this case the result caused by altering the parameters is more obvious and we can see the effect of waveguide adjustments easily. Reduce the length of the cavity to $10 \mathrm{~mm}$ that we actually use then we can see the effect of waveguide adjustments is limited, frequency of TM110 mode has a variation of about only $150 \mathrm{kHz}$ while length of the waveguides increases $10 \mathrm{~mm}$, so the effect of machining tolerance from waveguides is then limited, like Fig.5. In this case, the variation of the TM110 frequency caused by the change of the cavity radius is much larger, like Fig.6.

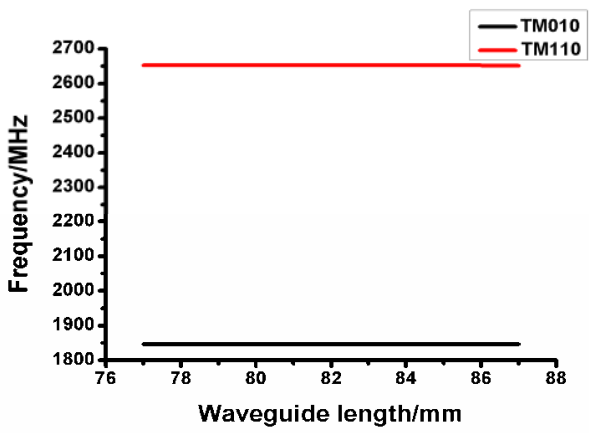

Figure 5: Effect of waveguide length

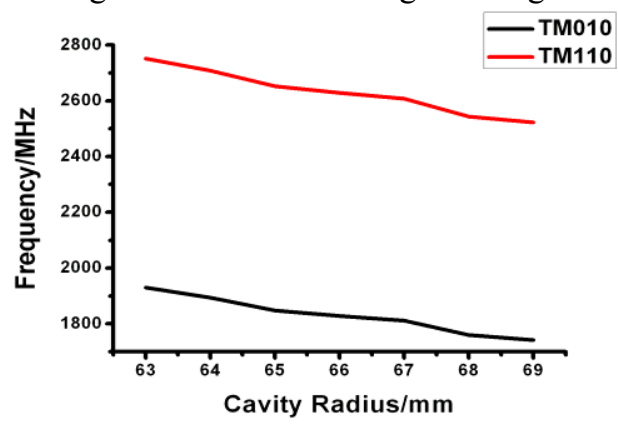

Figure 6: Effect of cavity radius

\section{REFERENCE CAVITY}

Table 3 shows the parameters of reference cavity. The equation (1) ${ }^{[2]}$ shows the relation between output signal and beam offset:

$$
V_{c a v}\left(\omega_{110}\right)=A_{1} q x+j A_{2} q+j A_{3} q x^{\prime}+V_{n}
$$

So we need a reference signal to normalize the signal and give the initial phase of the resonance field ${ }^{[2]}$. To 
provide the signal we use reference cavity. TM010 mode in reference cavity will be tuned to a working frequency at $2652 \mathrm{MHz}$. The TM010 signal from reference cavity is proportional to the beam current and will be used to normalize the position signal from position cavity.

Table 3: Parameters of the reference cavity

\begin{tabular}{|l|l|}
\hline Radius & $47.7 \mathrm{~mm}$ \\
\hline Length & $10.0 \mathrm{~mm}$ \\
\hline Frequency of TM010 mode & $2653.9 \mathrm{MHz}$ \\
\hline Q 0 of TM010 mode & 6682.8 \\
\hline
\end{tabular}

\section{ELECTRONICS}

The usual method is to use superheterodyne receivers in electronics for many cavity BPMs. Obviously we can use circuit chips instead of superheterodyne receivers to complete the process and simplify our work. Chip AD8302 from Analog Devices bases on the logarithmic compression function of logarithmic amplifier and uses two broadband logarithmic detectors that matches well to measure the magnitude and phase of the signals from two input channels. The AD8302 is a fully integrated system for measuring gain/loss and phase in numerous receive, transmit, and instrumentation applications. The input signals can range from $-60 \mathrm{dBm}$ to $0 \mathrm{dBm}$ in a $50 \Omega$ system, from low frequencies up to $2.7 \mathrm{GHz}$. The outputs provide an accurate measurement of either gain or loss over a $\pm 30 \mathrm{~dB}$ range scaled to $30 \mathrm{mV} / \mathrm{dB}$, and of phase over a $0^{\circ}-180^{\circ}$ range scaled to $10 \mathrm{mV} /$ degree $^{[9]}$. The block diagram of cavity-BPM detection circuit is shown in Fig.7. AD8302 will give the magnitude and phase signals. We can use computer to process them and then figure out the exact position of the beam. By using the chip AD8302 we can get an electronic system with more dynamic range and higher precision. Using the logarithmic RF amplifier, the signal processing system has large dynamic range.

\section{SUMMARY}

We have designed the prototype cavity BPM working at TM110 mode, $2652.0 \mathrm{MHz}$. Application of waveguides as pickups will suppress the interference from TM010 mode of resonance field. Use chip AD8302 to simplify the signal processing is a novel design in some way and we hope it will be of benefit to dynamic range and precision of electronics. The cavity beam position monitor system will help to improve the performance of HLS beam diagnostics system. Further work will be concentrated on the manufacture of the prototype cavity BPM and the signal processing system using AD8302.

\section{REFERENCES}

[1] J.H. Li, B.G. Sun, et al, "Research and Measurement of BPM System at HLS LINAC", High Energy Physics and Nuclear, 2007, 31 (01), p. 96.

[2] V. Sargsyan, "Cavity Beam Position Monitor for the TESLA-cryomodule. Cross-Talk minimization", Berlin, 2003.

[3] V. Sargsyan, "Comparison of Stripline and Cavity Beam Position Monitors", TESLA Report 2004-03

[4] R. Johnson, et al, "Cavity BPMs for the NLC", SLAC-PUB-9211, 2002.6.

[5] R. Lorenz, "Cavity Beam Position Monitors", DESY Zeuthen, Platanenallee 6, D-15738 Zeuthen.

[6] Z.H. Li, R. Johnson, S.R. Smith, et al, "Cavity BPM with Dipole-Mode-Selective Coupler", SLAC-PUB-11913, 2006.6.

[7] R. Ursic, R. Flood, et al, "1nA Beam Position Monitoring System", Thomas Jefferson National Accelerator Facility, Newport News, VA 23693, USA.

[8] A. Liapine, "Cavity Beam Position Monitor for the TESLA Energy Spectrometer", Proceedings DIPAC 2003-Mainz, Germany, 2003, p.184.

[9] Analog Devices, AD8302 Data Sheet, p.1.

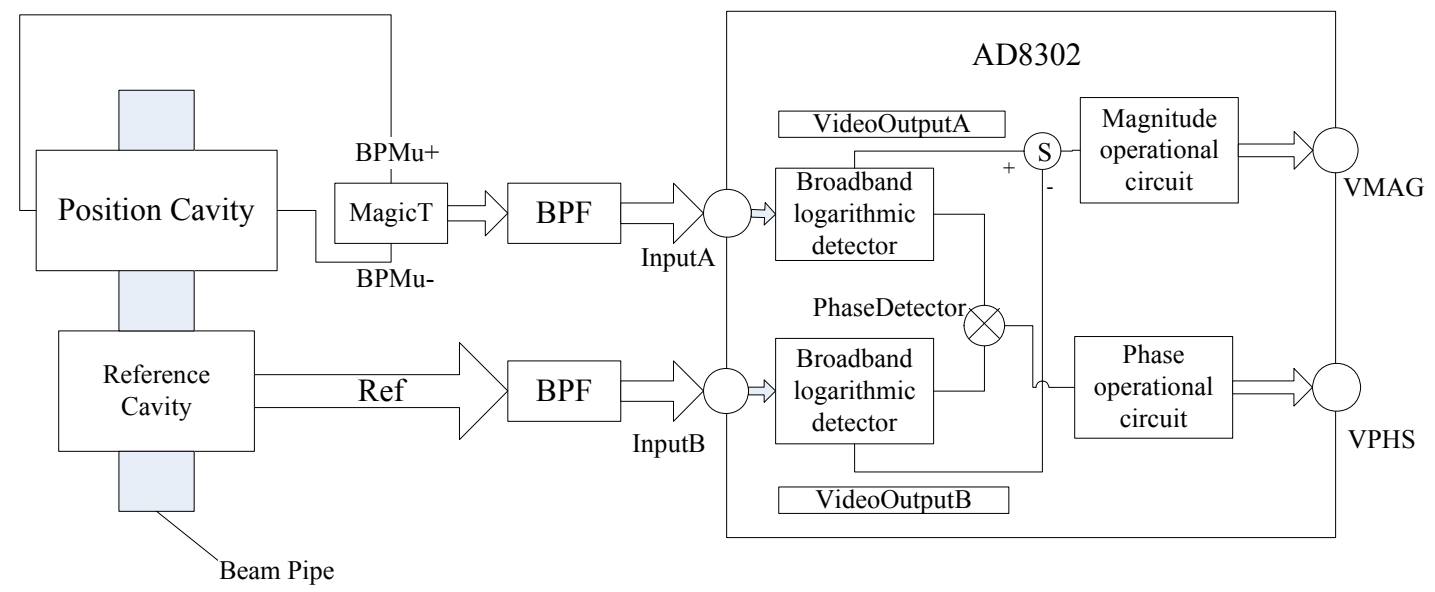

Figure 7: Block diagram of cavity-BPM detection circuit with AD8302 